\title{
Ultrasound-targeted microbubble destruction of calcium channel subunit $\alpha$ 1D siRNA inhibits breast cancer via $G$ protein-coupled receptor 30
}

\author{
YANLEI JI $^{1}$, ZHEN HAN ${ }^{2}$, LIMEI SHAO ${ }^{1}$ and YUEHUAN ZHAO ${ }^{1}$ \\ ${ }^{1}$ Department of Special Diagnosis, Shandong Cancer Hospital Affiliated to Shandong University, \\ Shandong Academy of Medical Sciences, Jinan, Shandong 250117; \\ ${ }^{2}$ Department of Internal Medicine, Jinan Second People's Hospital, Jinan, Shandong 250001, P.R. China
}

Received April 6, 2016; Accepted August 16, 2016

DOI: 10.3892/or.2016.5031

\begin{abstract}
Estrogen has been closely associated with breast cancer. Several studies reported that $\mathrm{Ca}^{2+}$ signal and $\mathrm{Ca}^{2+}$ channels act in estrogen-modulated non-genomic pathway of breast cancer, however little was revealed on the function of L-type $\mathrm{Ca}^{2+}$ channels. The L-type $\mathrm{Ca}^{2+}$ channel subunit $\alpha 1 \mathrm{D}$, named Cav1.3 was found in breast cancer cells. We aimed to investigate the expression and activity of Cav1.3 in human breast cancer, and reveal the effect of estrogen in regulating the expression of Cav1.3. The qRT-PCR and western blotting were employed to show that Cav1.3 was highly expressed in breast cancer tissues. E2 exposure rapidly upregulated the expression of Cav1.3 in dosage- and time-dependent manner, and promoted $\mathrm{Ca}^{2+}$ influx. The silencing of $\mathrm{G}$ protein-coupled estrogen receptor 30 (GPER1/GPR30) using siRNA transfection inhibited the upregulation of Cav1.3 and $\mathrm{Ca}^{2+}$ influx induced by E2. Moreover, the inhibition of Cav1.3 by siRNA transfection suppressed E2-induced second peak of $\mathrm{Ca}^{2+}$ signal, the expression of $\mathrm{p}-\mathrm{ERK} 1 / 2$, and the cell proliferation. Ultrasound-targeted microbubble destruction (UTMD) of Cav1.3 siRNA was used in MCF-7 cells in vitro and in the tumor xenografts mice in vivo. The application of UTMD significantly suppressed the tumor growth and promoted the survival rate. In conclusion, E2 upregulated the expression of Cav1.3 for $\mathrm{Ca}^{2+}$ influx to promote the expression of $\mathrm{p}$-ERK1/2 for cell proliferation. The study confirmed that the mechanism of E2 inducing the expression of Cav1.3 through a non-genomic pathway, and highlighted that UTMD of Cav1.3 siRNA is a powerful promising technology for breast cancer gene therapy.
\end{abstract}

Correspondence to: Dr Yuehuan Zhao, Department of Special Diagnosis, Shandong Cancer Hospital Affiliated to Shandong University, Shandong Academy of Medical Sciences, Jinan, Shandong 250117, P.R. China

E-mail: buea76449947@126.com

Key words: estrogen, non-genomic pathway, GPR30, Cav1.3, ultrasound-targeted microbubble destruction

\section{Introduction}

Normal growth and differentiation of the breast are largely known to be under endocrine control. The sex steroid hormone, estrogen, participates in the growth of normal breast tissue (1). Estrogen exerts its biological effects through two different pathways. On the one hand, estrogen could combine to the classical estrogen receptors $(\mathrm{ER} \alpha$ and $\mathrm{ER} \beta)$ to regulate target gene expression for the downstream cascades (2). In addition, estrogen could bind to the $\mathrm{G}$ protein-coupled estrogen receptor 30 (GPER1/GPR30) on the membrane with high affinity to mediate non-genomic responses (3). The imbalance of estrogen results in the occurrence of breast cancer (4). Breast cancer is one of the most common female cancers (5), and several studies have shown the close relationship between estrogen-activated non-genomic signaling and breast cancer. The membrane receptor GPR30 is widely expressed in breast cancer lines and breast primary tumors (6), and it is involved in estrogen-induced cell proliferation and migration (7).

Estrogen-induced non-genomic responses include cyclicAMP (cAMP) generation, intracellular calcium mobilization and mitogen-activated protein kinase (MAPK). $\mathrm{Ca}^{2+}$ homeostasis is linked with the modulation of $\mathrm{Ca}^{2+}, \mathrm{Ca}^{2+}$ channels and $\mathrm{Ca}^{2+}$-related proteins. In vascular smooth muscle cells, L-type $\mathrm{Ca}^{2+}$ channel blocker reduced intracellular $\mathrm{Ca}^{2+}$ induced by $17 \beta$-estradiol through GPR30 (8). 17 $\beta$-estradiol-induced $\mathrm{Ca}^{2+}$ influx via L-type calcium channels activates the Src/ERK/cAMP signal pathway in rat neurons (9). These findings show that $\mathrm{Ca}^{2+}$ channels have attracted interest, particularly L-type $\mathrm{Ca}^{2+}$ channels in estrogen signaling pathway. The L-type $\mathrm{Ca}^{2+}$ channel $\alpha$ 1D subunit Cav1.3 was identified and belongs to voltage-gated $\mathrm{Ca}^{2+}$ channels. Cav1.3 is highly expressed in prostate cancer tissues, and induces androgen-mediated cell growth mediated (10). In endometrial cancer cells, estrogen regulates Cav1.3 expression through GPR30, and initiates calcium influx via Cav1.3 to promote cell proliferation and migration by activating the ERK1/2/ CREB pathway (10). However, the function of Cav1.3 in breast cancer is not clear. In the present study, we investigated the mechanism by which estrogen regulates Cav1.3 expression and indicated the roles of Cav1.3 in breast cancer. 
$\mathrm{Ca}^{2+}$ channels are important therapeutic targets. Gene therapy using RNA interference can be directed against tumors, however, the enhancement of the transfection efficiency and specificity would be an important step for small interfering RNA (siRNA) delivery. To date ultrasound-targeted microbubble destruction (UTMD) is shown to be a valid method for gene transfection (11). In the present study, we investigated the effect of UTMD of Cav1.3 siRNA on tumor size and survival rate in breast cancer.

In the present study, we found that estrogen upregulated Cav1.3 expression in dosage- and time-dependent manner. Estrogen induced the calcium influx through GPR30 and Cav1.3. The increase of intracellular $\mathrm{Ca}^{2+}$ promoted the expression of $\mathrm{p}$-ERK1/2 to enhance cell proliferation. UTMD of Cav1.3 siRNA resulted in the inhibition of tumor volume and the increase of survival rate.

\section{Materials and methods}

Cell culture and transfections. Human breast cancer cell line MCF-7 was obtained from laboratory stocks and cultured in Dulbecco's modified Eagle's medium (DMEM), supplemented with $10 \%$ fetal bovine serum (both from Gibco-Invitrogen, Carlsbad, CA, USA) at $37^{\circ} \mathrm{C}$ in humidified incubator with $5 \%$ $\mathrm{CO}_{2}$. The siRNA sequences against Cav1.3, GPR30 and GFP were commercially obtained (HanBio, Shanghai, China).

MCF-7 cells were seeded into 6-well plates at 50\% confluency. Cav1.3 siRNA, GPR30 siRNA or GFP siRNA (Santa Cruz Biotechnology, Santa Cruz, CA, USA) was diluted into $250 \mu 1$ Opti-MEM medium at the concentration of $50 \mathrm{nM}$, and $5 \mu \mathrm{l}$ Lipofectamine 2000 (Invitrogen) was added into $250 \mu \mathrm{l}$ Opti-MEM medium at room temperature. After $10 \mathrm{~min}$, diluted siRNA and Lipofectamine 2000 were mixed well for another $10 \mathrm{~min}$, and then dispensed into plates. Fresh medium was added $6 \mathrm{~h}$ after transfection, and experiments were conducted for $48 \mathrm{~h}$ after transfection.

Western blotting. Proteins were isolated from the cells administered different treatments. Thirty micrograms protein was subjected to SDS-PAGE and transfected into polyvinylidene fluoride (PVDF) membranes (Millipore, Bedford, MA, USA). The membranes were blocked in $2 \%$ non-fat milk in Trisbuffered saline (TBS) (20 mM Tris and $140 \mathrm{mM} \mathrm{NaCl}$; pH 7.5) at room temperature, rinsed three times with $\mathrm{TBS}+0.2 \%$ Tween-20 (TBST), then incubated with Cav1.3 (1:1,000 dilution) or phospho-ERK1/2 (1:1,000 dilution) antibody overnight at $4^{\circ} \mathrm{C}$, followed by horseradish peroxidase-conjugated secondary antibody. The ECL substrate was used to detect their expression. The band intensities were determined using the Bio-Rad imaging system (Hercules, CA, USA).

Calcium measurements. MCF-7 cells were seeded in glass bottom fluorescence measurement dishes (Corning, Corning, NY, USA), and then pretreated with Cav1.3 siRNA, GPR30 siRNA or GFP siRNA for $48 \mathrm{~h}$, respectively. After being loaded with $10 \mu \mathrm{M}$ Fluo-3/AM (Invitrogen), cells were bathed in medium until treatment with E2 for $15 \mathrm{~min}$. The concentration of intracellular calcium was measured using a confocal laser scanning microscope Carl Zeiss LSM 700 (Thornwood, NY, USA). Data were analyzed using the Image-Pro Plus software.
5-Ethynyl-2'-deoxyuridine (EdU) detection. Cell proliferation was measured by EdU assay kit (Invitrogen). Cells were seeded into 96-well plates and transfected with Cav1.3 siRNA or GFP siRNA, respectively. After $48 \mathrm{~h}$, cells were exposed to $50 \mu \mathrm{M}$ of EdU for $4 \mathrm{~h}$ at $37^{\circ} \mathrm{C}$ and fixed in $4 \%$ paraformaldehyde for $10 \mathrm{~min}$ at room temperature. After being washed with a phosphate-buffered saline (PBS; $140 \mathrm{mM} \mathrm{NaCl}, 2.7 \mathrm{mM} \mathrm{KCl}$,

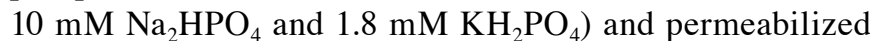
with $0.2 \%$ Triton $\mathrm{X}-100$ in $\mathrm{PBS}$ at $37^{\circ} \mathrm{C}$ for $30 \mathrm{~min}$ at room temperature. After washing with PBS twice for 5 min, cells were reacted with $100 \mu \mathrm{l}$ of $1 \mathrm{X}$ Apollo reaction cocktail for $30 \mathrm{~min}$, and were stained with $100 \mu \mathrm{l}$ of Hoechst $33342(5 \mu \mathrm{g} /$ $\mathrm{ml}$ ) for $30 \mathrm{~min}$. The nuclear was stained with 4',6-diamidino2-phenylindole (DAPI) $(10 \mathrm{mg} / \mathrm{ml})$ and visualized under a fluorescent microscope.

siRNA-microbubble preparation. Cationic lipid microbubbles were prepared by sonicating an aqueous dispersion of $1 \mathrm{mg} / \mathrm{ml}$ polyethylene glycol 2000 stearate (PEG2000), $2 \mathrm{mg} / \mathrm{ml}$ distearoylphophatidyl choline (DSPC) and $0.4 \mathrm{mg} / \mathrm{ml}$ 1,2-distearoyl-3-trimethyl ammonium propane (DOTAP) (all from Avanti, Germany) with perfluoropropane gas (12). Cav1.3 siRNA was added into cationic lipid microbubbles, and the mixture was incubated on a flat rocker to facilitate siRNA-microbubble interaction for $30 \mathrm{~min}$. MCF-7 cells were transfected with Cav1.3 siRNA-microbubbles in combination with ultrasound.

Tumor xenografting and ultrasound. Female athymic BALB/c nude mice (4-6 weeks old) were purchased from Shanghai Experimental Animal Centre, Chinese Academy of Science. For investigation in vivo, breast tumor xenografts were obtained by subcutaneously injecting $4 \times 10^{6} \mathrm{MCF}-7$ cells suspended in 0.2 $\mathrm{ml} 0.9 \% \mathrm{NaCl}$ into the nude mice. Once palpable tumors were established and reached $190 \mathrm{~mm}^{3}$, the mice were divided into groups for different treatments. G1, the group injected with 0.2 $\mathrm{ml} 0.9 \% \mathrm{NaCl}$; $\mathrm{G} 2$, the group injected with GFP siRNA; G3, the group injected with Cav1.3 siRNA; G4, the group injected with Cav1.3 siRNA-microbubbles. After being injected, mice were treated with ultrasound for $20 \mathrm{~min}$. A single-element transducer with a 1/2-inch diameter aperture with $1 \mathrm{MHz}$ ultrasound was used in the experiments. An acoustic pressure of $1 \mathrm{MPa}$ at the focus with a $50 \%$ duty cycle and a sonication intensity of $0.9 \mathrm{w} /$ $\mathrm{cm}^{2}$ were employed. The animal study was approved by the Ethics Committee of Shandong University.

Statistical analysis. The results are expressed as mean \pm SD. Means of different treatment groups were tested for statistical difference compared to the untreated control group using a Student's t-test and considered significantly different at $\mathrm{p}<0.05$. Statistical analysis was performed using Prism 5 (GraphPad Software, La Jolla, CA, USA).

\section{Results}

Cav1.3 is highly expressed in breast cancer tissues. It has been reported that Cav1.3 functions in tumor development $(10,13)$. We analyzed the expression of Cav1.3 in benign lesion breast and breast cancer tissues by qRT-PCR and western blotting. The mRNA level of Cav1.3 in breast cancer tissues 
A

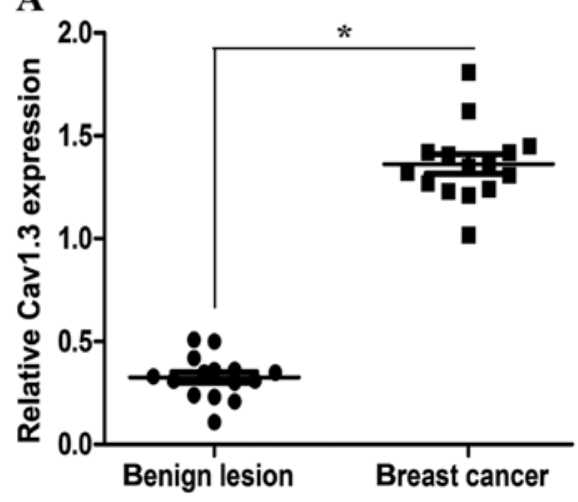

B
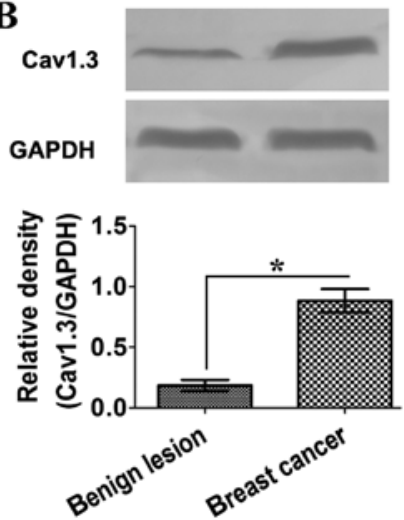

Figure 1. Cav1.3 is highly expressed in breast cancer tissues. Cav1.3 was overexpressed in the breast cancer tissues in the (A) mRNA and (B) protein levels. The mRNA and proteins from benign lesion breast tissues or breast cancer tissues were isolated and analyzed by qRT-PCR and western blotting. GAPDH was used as the interval control. Benign lesion, benign lesion breast tissues; breast cancer, breast cancer tissues; ${ }^{*} \mathrm{p}<0.05$.

A

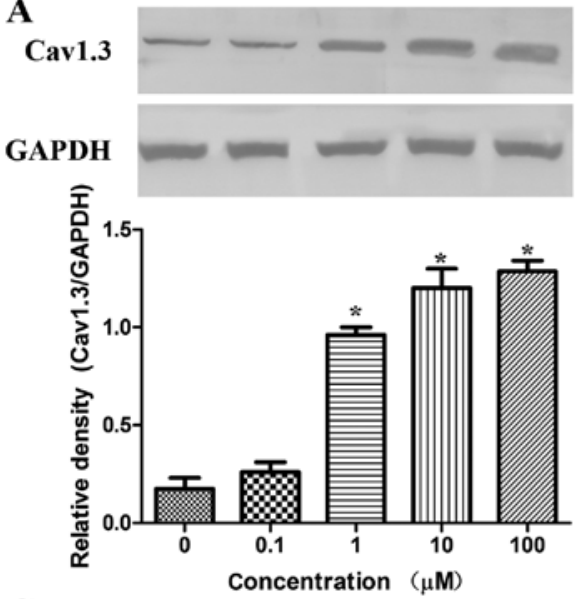

C
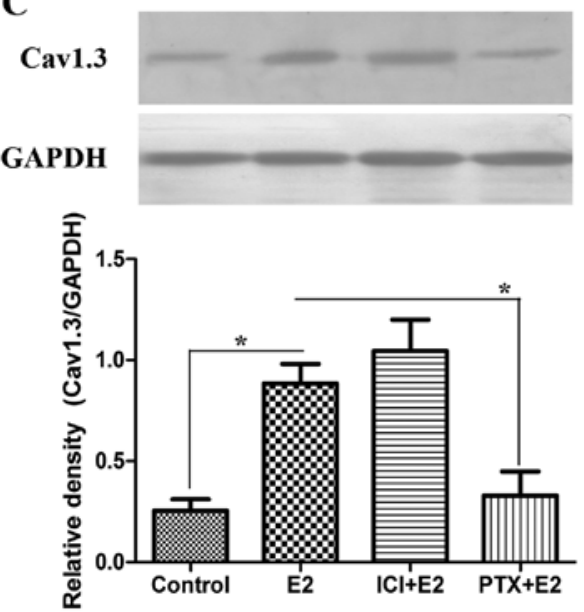

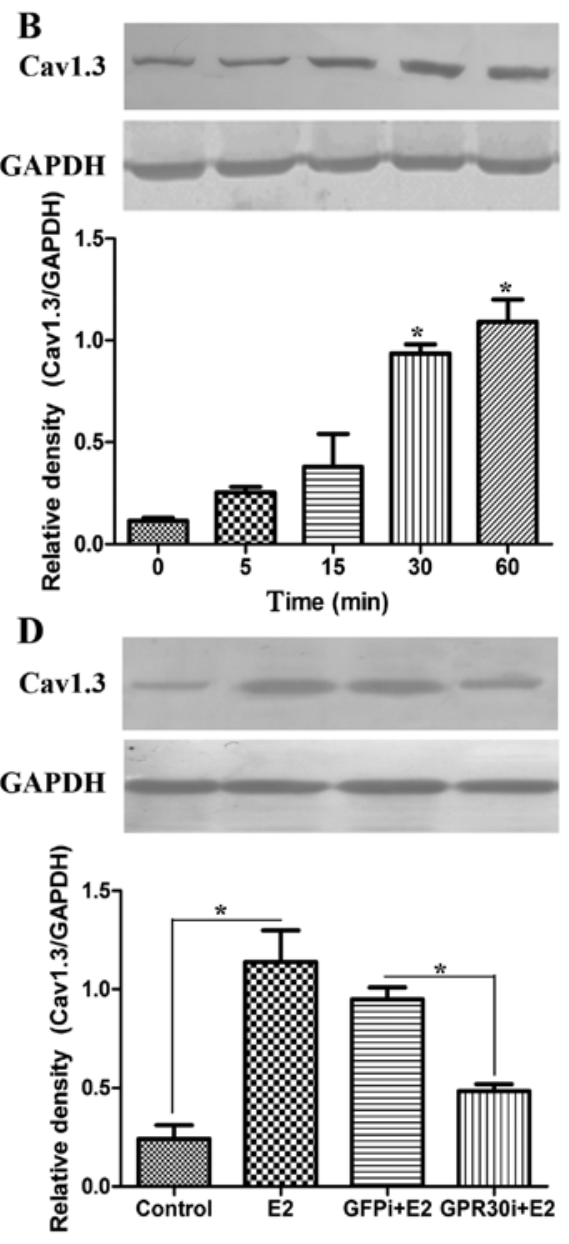

Figure 2. E2 upregulates the expression of Cav1.3 through GPR30 in MCF-7 cells. (A) E2 upregulated Cav1.3 expression in dosage-dependent manner analyzed by western blotting. MCF-7 cells were treated with E2 at the concentration of $0,0.1,1,10$ and $100 \mu \mathrm{M}$ for $1 \mathrm{~h}$, respectively. (B) Cav1.3 expression rapidly responded to E2 within $30 \mathrm{~min}$. MCF-7 cells were incubated with $1 \mu \mathrm{M} \mathrm{E} 2$ for 0, 5, 15, 30 and $60 \mathrm{~min}$, respectively. The proteins were extracted for western blotting with Cav1.3 antibody. GAPDH was used as the internal control; " $\mathrm{p}<0.05$. (C) The GPCR antagonist PTX suppressed the expression of Cav1.3 upregulated by E2. MCF-7 cells were pretreated with ER antagonist ICI $182780(1 \mu \mathrm{M})$ or GPCR antagonist PTX ( $200 \mathrm{ng} / \mathrm{ml})$, respectively. After $30 \mathrm{~min}$, cells were incubated with E2 at $1 \mu \mathrm{M}$ for $30 \mathrm{~min}$. Proteins were extracted for western blotting. (D) GPR30 siRNA inhibited the induction of Cav1.3 by E2. MCF-7 cells were transfected with GFP siRNA or GPR30 siRNA for $48 \mathrm{~h}$, respectively. After $1 \mu \mathrm{M}$ E2 incubation, proteins of different treatments were extracted for western blotting. GAPDH was used as the interval control; " $\mathrm{p}<0.05$.

was significantly higher than that in benign lesion breast tissues (Fig. 1A). Similar results were seen in the protein level (Fig. 1B). These results show that Cav1.3 may play a role in breast cancer development. 

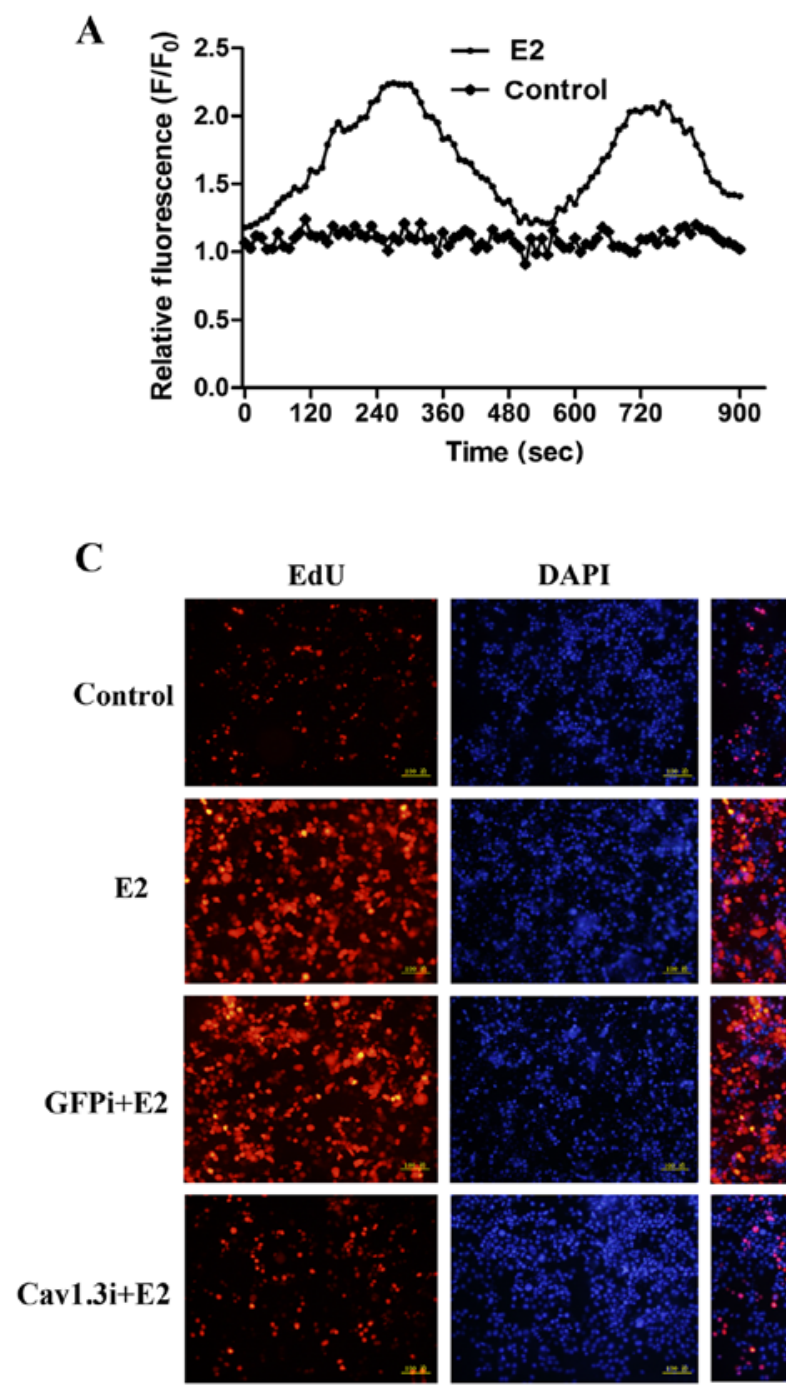
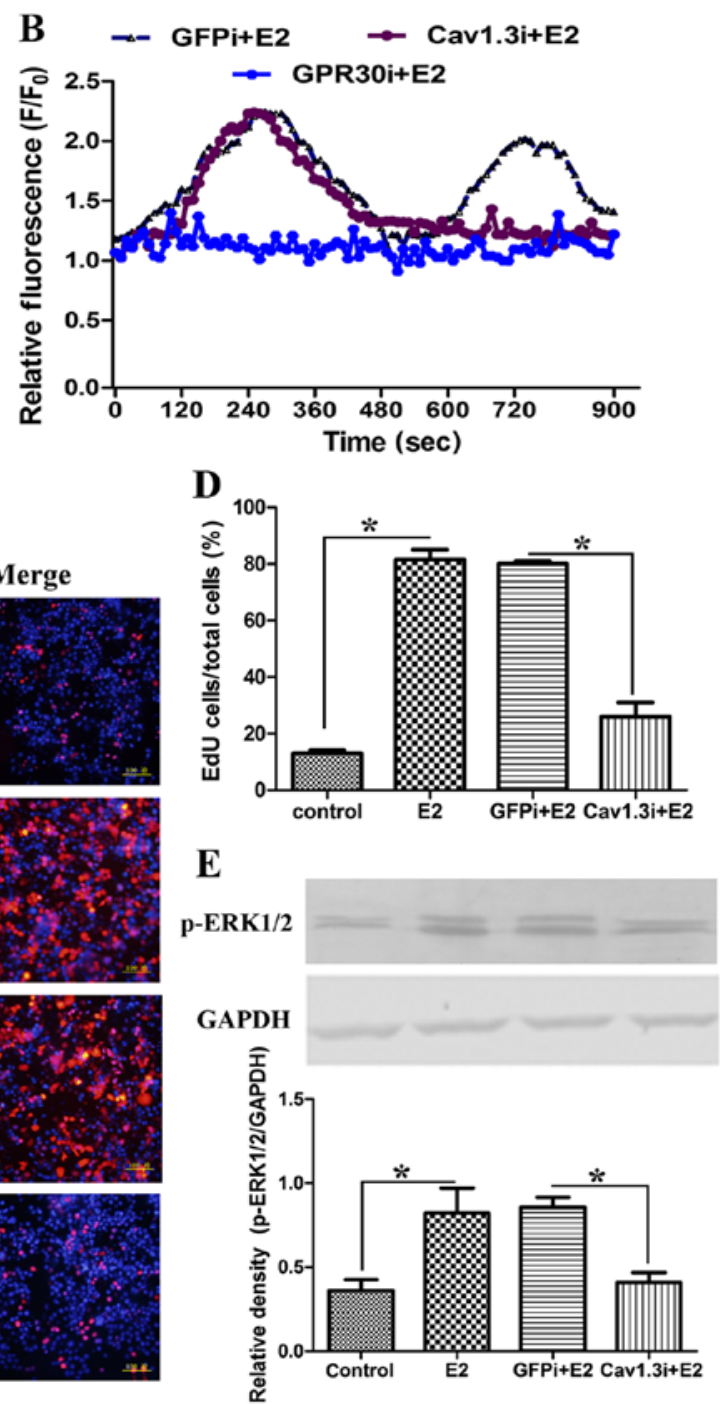

Figure 3. E2 promotes the cell proliferation through Cav1.3/ $\mathrm{Ca}^{2+} / \mathrm{p}-\mathrm{ERK} 1 / 2$ signaling pathway in MCF-7 cells. (A) E2 increased the level of intracellular Ca ${ }^{2+}$ determined by laser scanning confocal microscope. Cells were pretreated with $10 \mu \mathrm{M}$ Fluo-3/AM for $30 \mathrm{~min}$. After washing, cells were treated with $1 \mu \mathrm{M}$ $\mathrm{E} 2$, and then the $\mathrm{Ca}^{2+}$ level was detected with confocal microscopy. (B) E2 induced the $\mathrm{Ca}^{2+}$ influx via Cav1.3. Cells were transfected with GPR30 siRNA or Cav1.3 siRNA for $48 \mathrm{~h}$. After being loaded with Fluo-3/AM, E2 was added and the signal of $\mathrm{Ca}^{2+}$ was detected. GFP siRNA was used as the internal control. (C) E2 regulated the cell proliferation through Cav1.3. Cells were transfected with Cav1.3 siRNA for $48 \mathrm{~h}$. After E2 induction, cells were stained with EdU. GFP siRNA was used as the internal control. Scale bar, $100 \mu \mathrm{M}$. (D) Is the statistical analysis of C. (E) The silencing of Cav1.3 suppressed the expression of p-ERK1/2. Cells were transfected with Cav1.3 siRNA or GFP siRNA, respectively. After E2 treatment, proteins were isolated for western blotting with p-ERK1/2 antibody. GAPDH was used as the control; ${ }^{*} \mathrm{p}<0.05$.

E2 upregulates Cav1.3 expression through GPR30 in MCF-7 cells. The progression of breast cancer is reported to be associated with estrogen (14). In order to examine the effect of estrogen on Cav1.3 expression, we treated MCF-7 cells at different concentration of $\mathrm{E} 2$ and analyzed the protein level of Cav1.3. E2 upregulated Cav1.3 expression gradually in dosage-dependent manner (Fig. 2A). A time-course experiment was performed for 0-60 min with $1 \mu \mathrm{M} \mathrm{E} 2$ chosen for subsequent analysis. Concerning the expression of Cav1.3, a distinct response was observed within $30 \mathrm{~min}$ (Fig. 2B). These results revealed that E2 may upregulate Cav1.3 expression through the membrane non-genomic pathway. We found GPCR antagonist PTX, but not ER antagonist ICI 182780 inhibited Cav1.3 expression in MCF-7 cells (Fig. 2C), showing that some GPCR is involved in estrogen-induced Cav1.3 expression. In endometrial cancer HEC-1A cells, estrogen modulated Cav1.3 expression rapidly through GPR30 (13). To confirm the function of GPR30, we knocked down GPR30 and examined the protein level of Cav1.3 after E2 induction. The silencing of GPR30 suppressed the expression of Cav1.3 (Fig. 2D). These results show that E2 upregulates Cav1.3 expression through GPR30 on the membrane in MCF-7 cells.

Estrogen modulates calcium mobilization and activated p-ERK1/2 expression via Cav1.3 to promote cell proliferation. In MCF-7 cells, we found estrogen stimulated the increase of intracellular $\mathrm{Ca}^{2+}$ signal. After E2 induction, the $\mathrm{Ca}^{2+}$ level showed two peaks. The first peak appeared at $280 \mathrm{sec}$, and the second at $720 \mathrm{sec}$ (Fig. 3A). When GPR30 was blocked, the two waves were inhibited (Fig. 3B). In order to investigate the effect of Cav1.3 on $\mathrm{Ca}^{2+}$ signal, we knocked down Cav1.3. The silencing of Cav1.3 significantly affected E2-induced 

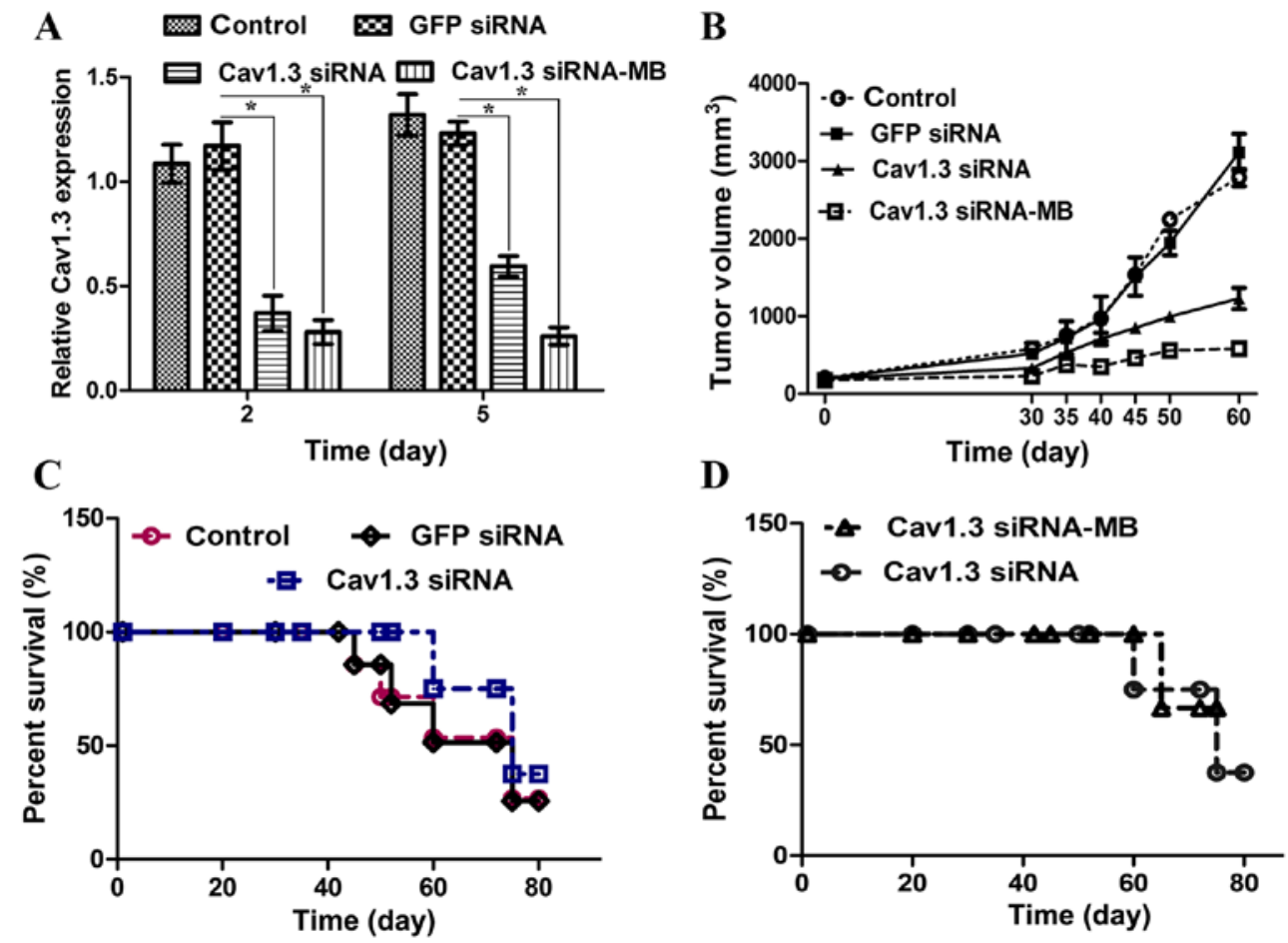

Figure 4. The silencing of Cav1.3 significantly suppresses the tumor growth and improves the survival rate. (A) Cav1.3 siRNA-MB prolonged the silencing time of Cav1.3. MCF-7 cells were transfected with Cav1.3 siRNA or Cav1.3 siRNA-MB with ultrasound. Approximately 48 or $120 \mathrm{~h}$ later, cells were treated with E2 and total RNA was isolated for qRT-PCR analysis. GFP siRNA was the control siRNA. GAPDH was used as the internal control. Human breast cancer xenografts were injected with Cav1.3 siRNA or Cav1.3 siRNA-MB, and then were treated with ultrasound for siRNA delivery. The tumor sizes and survival rate of mice with different treatments were determined. (B) UTMD of Cav1.3 siRNA suppressed the tumor growth. (C) Cav1.3 siRNA improved the survival rate. (D) UTMD of Cav1.3 siRNA increased the survival rate compared to Cav1.3 siRNA. Cav1.3 siRNA-MB, Cav1.3 siRNA-microbubbles; ${ }^{*}$ p $<0.05$.

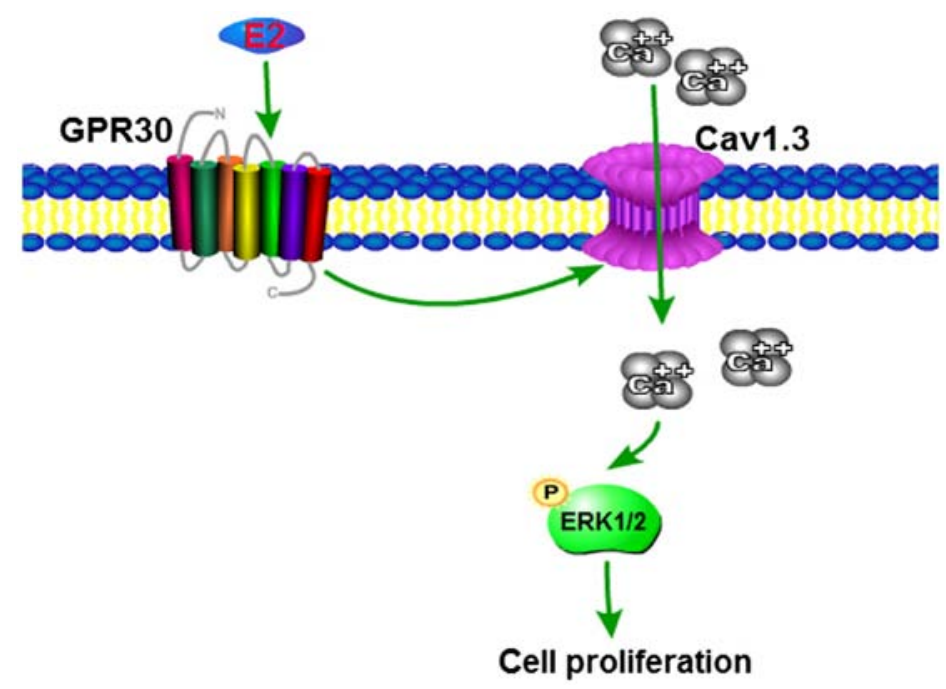

Figure 5. The signaling pathway initiated by estrogen in MCF-7 cells. Estrogen upregulated the expression of Cav1.3 through the membrane GPR30. The activation of Cav1.3 promoted the $\mathrm{Ca}^{2+}$ influx, then the increase of $\mathrm{Ca}^{2+}$ induced the expression of $\mathrm{p}$-ERK1/2 pathway to initiate the cell proliferation.

second peak (Fig. 3B), showing that Cav1.3 is involved in E2-stimulated $\mathrm{Ca}^{2+}$ influx. Various studies have shown that the $\mathrm{Ca}^{2+}$ signal is closely related to cell proliferation (15). Cell proliferation was detected by EdU staining to examine the role of Cav1.3. The silencing of Cav1.3 significantly blocked cell proliferation (Fig. 3C and D) and the expression of p-ERK1/2 (Fig. 3E). These results revealed that E2 induces $\mathrm{Ca}^{2+}$ influx via Cav1.3 to increase the expression of p-ERK1/2 and promote cell proliferation.
Cav1.3 siRNA-microbubble with ultrasound improves the interference efficiency. In order to determine whether or not UTMD is a good method for siRNA delivery, UTMD of Cav1.3 siRNA was used in MCF-7 cells in vitro and in mice in vivo. The time of Cav1.3 silencing by UTMD of Cav1.3 siRNA was longer than that only by Cav1.3 siRNA tranfection (Fig. 4A). Cav1.3 siRNA injection diminished the tumor volume. Moreover, the tumor size in the mice treated with Cav1.3 siRNA-microbubbles was smaller than that in the 
Cav1.3 siRNA injected mice (Fig. 4B). The Cav1.3 siRNA delivery increased the survival rate and delayed the day of first death (Fig. 4C). Compared with the Cav1.3 siRNA injection, the effect of Cav1.3 siRNA-microbubble delivery on tumor growth and survival rate was more significant (Fig. 4D). These results reveal that UTMD of Cav1.3 enhances the efficiency of RNA interference. The signaling pathway initiated by estrogen in MCF-7 cells is shown in Fig. 5.

\section{Discussion}

E2 rapidly upregulates Cav1.3 expression through GPR30 in MCF-7 cells. Cav1.3, as a type of L-type $\mathrm{Ca}^{2+}$ channel, exists in many tissues, including brain, nervous system and kidney. Although Cav1.3 is overexpressed in endometrial (13) and prostate cancers (10), the relationship between Cav1.3 and breast cancer is not clear. In the present study, Cav1.3 was highly expressed in the breast cancer tissues, revealing Cav1.3 functions in tumor development. E2 rapidly stimulated Cav1.3 expression within $30 \mathrm{~min}$ in MCF-7 cells. The ER antagonist ICI 182780 did not inhibit the upregulation of Cav1.3 induced by E2, but GPCR antagonist PTX did, indicating that E2 modulated the Cav1.3 expression ER-independently. It has been shown that E2-induced non-genomic pathway via the membrane receptor GPR30 is associated with tumor development (16). 17 $\beta$-estradiol induced migration, adhesion and invasion of breast cancer through GPR30 (17). The expression of GPR30 is prognostic in primary breast cancer (18). When GPR30 was knocked down, the activation of Cav1.3 was blocked, showing that E2 upregulated Cav1.3 expression via GPR30. These results indicated the function of Cav1.3 in breast cancer and showed a new mechanism by which estrogen regulated Cav1.3 expression.

$\mathrm{Ca}^{2+}$-mediated signaling plays roles in various cellular processes, including cancer initiation, tumor progression and invasion (19). E2 could either activate (20) or inhibit (21) $\mathrm{Ca}^{2+}$ influx via $\mathrm{Ca}^{2+}$ channels (22). In the present study, E2 induced two peak of $\mathrm{Ca}^{2+}$ at 280 and $720 \mathrm{sec}$ in MCF-7 cells. When Cav1.3 was silenced, the second wave was blocked, revealing that E2 induced $\mathrm{Ca}^{2+}$ flux through Cav1.3. Transfection of Cav1.3 siRNA suppressed the cell proliferation of MCF-7 cells and the expression of p-ERK1/2. It has been shown that E2 activated the ERK1/2/CREB signaling by the rapid $\mathrm{Ca}^{2+}$ influx via L-type $\mathrm{Ca}^{2+}$ channel (9), which provided a link between $\mathrm{Ca}^{2+}$ flux and gene transcription. In endometrial cancer, Cav1.3 was necessary for the expression of p-ERK1/2 and CREB (13). In addition, in MCF-7 cells, E2 induced $\mathrm{Ca}^{2+}$ influx via Cav1.3 to activate the expression of p-EKR1/2 to promote the cell proliferation. Taken together, these findings showed the mechanism of Cav1.3 involving in the progression of breast cancer.

Cav1.3 siRNA-MB delivered by ultrasound suppresses the tumor growth and improves the survival rate. The above results have revealed that Cav1.3 may be a new target for the treatment of breast cancer. SiRNAs have become useful tools to inactivate target gene expression, however the efforts on the delivery efficacy and specificity in the clinic need further attention (23). Various studies have shown that UTMD could be a powerful technology for gene therapy (24), including plasmid (25), siRNA (26) and different drugs delivery (27). In the present study, we found that the UTMD of Cav1.3 siRNA prolonged the time of Cav1.3 silencing than Cav1.3 siRNA, revealing that the microbubble of Cav1.3 siRNA maintained the stability of siRNA. In vitro, the tumor volume of the mice treated with the UTMD of Cav1.3 siRNA was significantly reduced, and the first death day of these mice was obviously delayed. Therefore, Cav1.3 siRNA in combination with UTMD enhanced the efficacy of gene therapy, and UTMD is a promising method for breast cancer therapy.

In conclusion, in the present study, Cav1.3 was highly expressed in breast cancer. E2 activated the expression of Cav1.3 via the membrane receptor GPR30 in MCF-7 cells. Moreover, E2 induced $\mathrm{Ca}^{2+}$ influx through Cav1.3 to activate the expression of $\mathrm{p}$-ERK1/2 for cell proliferation. These results revealed the biological basis of E2-inducing Cav1.3 expression and the function of Cav1.3 in breast cancer. We employed the UTMD method to deliver Cav1.3 siRNA into cells and mice to show that UTMD of Cav1.3 siRNA is a useful tool for breast cancer therapy.

\section{References}

1. Pelekanou V and Leclercq G: Recent insights into the effect of natural and environmental estrogens on mammary development and carcinogenesis. Int J Dev Biol 55: 869-878, 2011.

2. Gibson DA and Saunders PT: Estrogen dependent signaling in reproductive tissues - a role for estrogen receptors and estrogen related receptors. Mol Cell Endocrinol 348: 361-372, 2012.

3. Revankar CM, Cimino DF, Sklar LA, Arterburn JB and Prossnitz ER: A transmembrane intracellular estrogen receptor mediates rapid cell signaling. Science 307: 1625-1630, 2005.

4. Gompel A and Santen RJ: Hormone therapy and breast cancer risk 10 years after the WHI. Climacteric 15: 241-249, 2012.

5. Kamangar F, Dores GM and Anderson WF: Patterns of cancer incidence, mortality, and prevalence across five continents: Defining priorities to reduce cancer disparities in different geographic regions of the world. J Clin Oncol 24: 2137-2150, 2006.

6. Scaling AL, Prossnitz ER and Hathaway HJ: GPER mediates estrogen-induced signaling and proliferation in human breast epithelial cells and normal and malignant breast. Horm Cancer 5: 146-160, 2014.

7. Pandey DP, Lappano R, Albanito L, Madeo A, Maggiolini M and Picard D: Estrogenic GPR30 signalling induces proliferation and migration of breast cancer cells through CTGF. EMBO J 28: 523-532, 2009.

8. Holm A, Hellstrand P, Olde B, Svensson D, Leeb-Lundberg LM and Nilsson BO: The G protein-coupled estrogen receptor 1 (GPER1/GPR30) agonist G-1 regulates vascular smooth muscle cell $\mathrm{Ca}^{2+}$ handling. J Vasc Res 50: 421-429, 2013.

9. Wu TW, Wang JM, Chen S and Brinton RD: $7 \beta$-estradiol induced $\mathrm{Ca}^{2+}$ influx via L-type calcium channels activates the $\mathrm{Src} / \mathrm{ERK} /$ cyclic-AMP response element binding protein signal pathway and BCL-2 expression in rat hippocampal neurons: a potential initiation mechanism for estrogen-induced neuroprotection. Neuroscience 135: 59-72, 2005.

10. Chen R, Zeng X, Zhang R, Huang J, Kuang X, Yang J, Liu J, Tawfik O, Thrasher JB and Li B: Cav 1.3 channel $\alpha 1 \mathrm{D}$ protein is overexpressed and modulates androgen receptor transactivation in prostate cancers. In: Urologic Oncology: Seminars and Original Investigations. Vol 32. Issue 5. Elsevier, pp524-536, 2014.

11. Carson AR, McTiernan CF, Lavery L, Grata M, Leng X, Wang J, Chen $\mathrm{X}$ and Villanueva FS: Ultrasound-targeted microbubble destruction to deliver siRNA cancer therapy. Cancer Res 72: 6191-6199, 2012

12. Leong-Poi H, Kuliszewski MA, Lekas M, Sibbald M, Teichert-Kuliszewska K, Klibanov AL, Stewart DJ and Lindner JR: Therapeutic arteriogenesis by ultrasound-mediated VEGF $_{165}$ plasmid gene delivery to chronically ischemic skeletal muscle. Circ Res 101: 295-303, 2007. 
13. Hao J, Bao X, Jin B, Wang X, Mao Z, Li X, Wei L, Shen D and Wang JL: $\mathrm{Ca}^{2+}$ channel subunit $\alpha$ 1D promotes proliferation and migration of endometrial cancer cells mediated by $17 \beta$-estradiol via the $G$ protein-coupled estrogen receptor. FASEB J 29: 2883-2893, 2015.

14. Clemons M and Goss P: Estrogen and the risk of breast cancer. N Engl J Med 344: 276-285, 2001.

15. Hao B, Webb SE, Miller AL and Yue J: The role of $\mathrm{Ca}^{2+}$ signaling on the self-renewal and neural differentiation of embryonic stem cells (ESCs). Cell Calcium 59: 67-74, 2016.

16. Lappano R, Pisano A and Maggiolini M: GPER function in breast cancer: An overview. Front Endocrinol 5: 66, 2014.

17. Shang D, Li Z, Zhu Z, Chen H, Zhao L, Wang X and Chen Y: Baicalein suppresses 17- $\beta$-estradiol-induced migration, adhesion and invasion of breast cancer cells via the $G$ protein-coupled receptor 30 signaling pathway. Oncol Rep 33: 2077-2085, 2015.

18. Wanajo A, Sasaki A, Nagasaki H, Shimada S, Otsubo T, Owaki S, Shimizu Y, Eishi Y, Kojima K, Nakajima Y, et al: Methylation of the calcium channel-related gene, $C A C N A 2 D 3$, is frequent and a poor prognostic factor in gastric cancer. Gastroenterology 135 , 580-590, 2008

19. Prevarskaya N, Skryma R and Shuba Y: Calcium in tumour metastasis: New roles for known actors. Nat Rev Cancer 11: 609-618, 2011.

20. Sarkar SN, Huang RQ, Logan SM, Yi KD, Dillon GH and Simpkins JW: Estrogens directly potentiate neuronal L-type $\mathrm{Ca}^{2+}$ channels. Proc Natl Acad Sci USA 105: 15148-15153, 2008.
21. Boulware MI, Weick JP, Becklund BR, Kuo SP, Groth RD and Mermelstein PG: Estradiol activates group I and II metabotropic glutamate receptor signaling, leading to opposing influences on cAMP response element-binding protein. J Neurosci 25: 5066-5078, 2005.

22. Improta-Brears T, Whorton AR, Codazzi F, York JD, Meyer T and McDonnell DP: Estrogen-induced activation of mitogenactivated protein kinase requires mobilization of intracellular calcium. Proc Natl Acad Sci USA 96: 4686-4691, 1999.

23. Castanotto D and Rossi JJ: The promises and pitfalls of RNA-interference-based therapeutics. Nature 457: 426-433, 2009.

24. Cao WJ, Rosenblat JD, Roth NC, Kuliszewski MA, Matkar PN, Rudenko D, Liao C, Lee PJ and Leong-Poi H: Therapeutic angiogenesis by ultrasound-mediated microRNA-126-3p delivery. Arterioscler Thromb Vasc Biol 35: 2401-2411, 2015.

25. Taniyama Y, Tachibana K, Hiraoka K, Namba T, Yamasaki K, Hashiya N, Aoki M, Ogihara T, Yasufumi K and Morishita R: Local delivery of plasmid DNA into rat carotid artery using ultrasound. Circulation 105: 1233-1239, 2002.

26. Xu Q, Sun T, Tian H, Wang $\mathrm{C}$ and Zhou H: Ultrasound-mediated vascular endothelial growth factor C (VEGF-C) gene microbubble transfection inhibits growth of MCF-7 breast cancer cells. Oncol Res 20: 297-301, 2013.

27. Frenkel V: Ultrasound mediated delivery of drugs and genes to solid tumors. Adv Drug Deliv Rev 60: 1193-1208, 2008. 